\title{
Plasma fetuin-A concentrations in young and older high- and low-active men
}

\author{
Nathan T. Jenkins ${ }^{1,{ }^{*}, \text { Jennifer A. McKenzie }}{ }^{2,{ }^{*}, \text { James M. Hagberg }}{ }^{1}$, and Sarah Witkowski ${ }^{1}$ \\ ${ }^{1}$ Department of Kinesiology, School of Public Health, University of Maryland College Park, MD \\ ${ }^{2}$ Department of Exercise Science \& Physical Education, McDaniel College, Westminster, MD
}

\section{Abstract}

Fetuin-A is a liver-derived factor that may play a role in insulin resistance and age-related chronic diseases [e.g., type 2 diabetes and cardiovascular (CV) disease]. Regular exercise improves CV risk and insulin sensitivity, however it is unknown whether chronic exercise training is related to circulating levels of fetuin-A. Therefore, this study examined whether plasma fetuin-A levels were related to age and chronic physical activity in men. We hypothesized that chronic physical activity would be related to lower plasma fetuin-A levels in younger and older men. In healthy highlyactive (HI) and low-active (LO) young (HI: $\mathrm{n}=7$; LO: $\mathrm{n}=8$ ) and older (HI: $\mathrm{n}=12, \mathrm{LO}: \mathrm{n}=11$ ) men, we determined cardiorespiratory fitness $\left(\mathrm{VO}_{2} \mathrm{max}\right)$, plasma fetuin-A levels, plasma insulin, insulin resistance (HOMA-IR), and the standard risk factors for $\mathrm{CV}$ disease. Groups were matched for body mass index (BMI). Fetuin-A was significantly higher ( 20\%) in both young and older LO men compared to their $\mathrm{HI}$ counterparts, and fetuin- $\mathrm{A}$ was inversely related to $\mathrm{VO}_{2} \max (\mathrm{r}=$ $-0.40, P=0.014)$. Plasma fetuin-A levels showed trends to be significantly correlated with insulin $(r=-0.34, P=0.052)$ and HOMA-IR $(r=0.33, P=0.058)$ in the older individuals. In younger participants, fetuin-A was related to blood pressure and cholesterol measures. These results indicate that low levels of fetuin-A are related to cardiorespiratory fitness and a number of conventional $\mathrm{CV}$ and metabolic disease risk factors independent of age and BMI. Therefore, the maintenance of low levels of circulating fetuin-A may be a novel mechanism contributing to enhanced insulin sensitivity with regular physical activity.

\section{Keywords}

Fetuin-A; Exercise; Cardiovascular Disease; Diabetes; Insulin sensitivity

\section{Introduction}

Cardiovascular (CV) disease is the leading cause of death in developed countries and the vascular complications associated with dysregulated glucose metabolism make CV disease the major cause of death for people with Type 2 diabetes [1]. Insulin resistance is a precursor to diabetes [2]; however the factors influencing insulin resistance are not fully

Correspondence: Sarah Witkowski, Ph.D., Department of Kinesiology, 106 Totman Building, University of Massachusetts, 30

Eastman La., Amherst, MA 01003, switkows@kin.umass.edu.

Authors contributed equally.

The authors report no conflict of interest or financial disclosures.

Publisher's Disclaimer: This is a PDF file of an unedited manuscript that has been accepted for publication. As a service to our customers we are providing this early version of the manuscript. The manuscript will undergo copyediting, typesetting, and review of the resulting proof before it is published in its final citable form. Please note that during the production process errors may be discovered which could affect the content, and all legal disclaimers that apply to the journal pertain. 
understood. There is a need to identify novel risk factors for CV and metabolic disease, as some estimates indicate that conventional risk factors including hypertension, dyslipidemia, etc. explain only $50 \%$ of actual CV disease cases [3]. The effects of regular exercise on CV risk and glucose metabolism are well-characterized and include robust effects on skeletal muscle insulin action and glucose transport and suppression of hepatic glucose production [4]. However, here again there is a "risk factor gap", as physical activity effects on conventional risk factors explain only $\sim 60 \%$ of the actual reduction in risk of $\mathrm{CV}$ and metabolic disease through regular physical activity [5]. Therefore, there is a need to identify novel risk factors that contribute to $\mathrm{CV}$ and metabolic disease and analyze whether lifestyle variables, such as physical activity influence these factors [6].

Fetuin-A ( $\alpha 2$-Heremans-Schmid glycoprotein) is a liver-derived circulating protein and is a ligand for tyrosine kinase receptors. Early experiments in rodent models indicated that fetuin-A binding to insulin receptors in adipose tissue and skeletal muscle decreases receptor phosphorylation and reduces downstream insulin signaling [7]. More recently, several lines of evidence have indicated that fetuin-A is involved in CV and metabolic disease risk. Fetuin-A levels have been positively associated with diabetes risk in middle-aged and older individuals [8], incident diabetes in older men and women [9], carotid artery intima-media thickness in middle-aged adults [10], and risk of myocardial infarction and stroke [11]. In addition, polymorphic variation in the fetuin-A gene $(A H S G)$ has been associated with $\mathrm{CV}$ diseases in a European population [12].

It is largely unknown if chronic physical activity influences circulating levels of fetuin-A and whether age may play a role in this relationship. As a possible biological link between physical activity and fetuin-A levels, it is known that cardiorespiratory fitness is inversely associated with liver fat [13], and fetuin-A is associated with liver fat accumulation in humans [14,15]. Further, Kantartzis et al. [16] found that high cardiorespiratory fitness was a strong independent predictor of the decrease in liver fat following a combined exercise and dietary lifestyle intervention in non-alcoholic fatty liver disease patients. In a comprehensive investigation into the relationships between fetuin- $\mathrm{A}$ and $\mathrm{CV} /$ metabolic dysregulation [17], fetuin-A levels were positively correlated with C-reactive protein and negatively correlated with adiponectin levels, indicating a link between elevated fetuin-A levels and a proinflammatory state. Further mechanistic experiments revealed that exogenous fetuin-A treatment increased expression of inflammatory genes (TNF- $\alpha$ and interleukin-1 $\beta$ ) and reduced adiponectin gene expression in vitro (cultured human adipocytes and macrophages) and in vivo (mouse adipose tissue) [17]. Together, these data suggest that fetuin-A induces low grade inflammation, a well known hallmark of habitual sedentary behavior that is readily improved by endurance exercise training [18].

Therefore, the purpose of this study was to determine whether plasma fetuin-A levels were related to physical activity in young and older healthy men. We hypothesized that low cardiorespiratory fitness and older age would be associated with elevated fetuin-A levels. We also examined the relationships between fetuin-A levels and a number of conventional $\mathrm{CV}$ and metabolic risk factors.

\section{Methods}

\subsection{Screening}

All screening, recruiting, and testing methods for the subjects in this study were described previously $[19,20]$. Briefly, participants were healthy, non-smoking, non-diabetic men with no history of CV disease and were recruited on the basis of age, BMI, and physical activity level. A total of 19 highly-active men (HI) and 19 low-active men (LO) participated in the study. The young active (young HI, $n=7$ ) group consisted of men aged 18-30 who had 
engaged in $>3$ years of moderate to intense physical activity $>4 \mathrm{hr} /$ week. Older active (older HI) men $(n=12$, aged 55-80) had a $>20$ year history of endurance physical activity $>3$ days/week. The low-active ( $\leq 20 \mathrm{~min} /$ day, $\leq 2$ days/week, young LO and older LO, $\mathrm{n}=8$ and $n=11$, respectively) groups were matched to HI for age and BMI. Written informed consent was obtained from all participants and all study procedures were approved by the University of Maryland College Park Institutional Review Board.

\subsection{Maximal oxygen uptake $\left(\mathrm{VO}_{2} \mathrm{max}\right)$ and body composition}

$\mathrm{VO}_{2}$ max and body composition were determined as described in our previous studies $[19,20]$.

\subsection{Plasma variables}

Blood was sampled at rest following an overnight fast for blood chemistry profile (Quest Diagnostics), plasma insulin, and fetuin-A. Plasma insulin was assayed using a commercially-available ELISA kit (Alpco, Salem, NH). Intra- and inter-assay coefficients of variation were $10 \%$ and $11.3 \%$, respectively. The homeostasis model assessment estimate of insulin resistance (HOMA-IR) was calculated using plasma insulin and glucose concentrations according to Matthews et al. [21]. Plasma fetuin-A was determined by ELISA (Human Fetuin-A ELISA Kit, Epitope Diagnostics, Inc, San Diego, CA). Intra- and inter-assay coefficients of variation were $6.5 \%$ and $12.2 \%$, respectively.

\subsection{Statistics}

Data are presented as means \pm SE. Pearson correlation coefficients were used to analyze relationships between variables for all participants and for groups separated by age. Multiple regression was used to examine associations between standard $\mathrm{CV}$ risk factors and fetuin-A levels in the entire study sample with age and BMI as covariates. Differences among all four study groups were analyzed via ANOVA, and the difference between young and older groups combined was analyzed by an unpaired t-test. An $\alpha$ level of 0.05 was used to indicate statistical significance.

\section{Results}

\subsection{Subject characteristics}

Subject characteristics are shown in Table 1 . There were no statistically significant differences among the four groups in BMI, and $\mathrm{HI}$ and $\mathrm{LO}$ within each age category were generally matched for the standard $\mathrm{CV}$ disease risk factor profile. $\mathrm{HI}$ and $\mathrm{LO}$ differed substantially in $\mathrm{VO}_{2}$ max within each age group. Therefore, our efforts to recruit solely on the basis of age, BMI, and training status were successful. In addition, the groups were, in general, well matched on the conventional CV risk factor variables (Table 1).

\subsection{Fetuin-A levels}

There was a main effect of training status on fetuin-A levels $(\mathrm{P}<0.05)$, with no main effect of age and no age $\times$ training status interactive effect (Figure 1A). A significant inverse correlation was found between $\mathrm{VO}_{2}$ max and plasma fetuin-A levels across all study subjects $(\mathrm{r}=-0.40, \mathrm{P}=0.01)$ (Figure 1B). Age groups did not differ in fetuin-A levels $(\mathrm{P}=0.68$, Figure 1C). In an additional statistical approach examining the association between fetuin-A levels and conventional $\mathrm{CV}$ risk factors with adjustments for age and BMI, the correlation between fetuin- $\mathrm{A}$ and $\mathrm{VO}_{2}$ max remained statistically significant $(\mathrm{r}=-0.48, \mathrm{P}=0.003)$ and neither age nor BMI significantly contributed to the variance in fetuin-A levels (both $\mathrm{P}>$ 0.05). There were no other statistically significant correlations between fetuin-A levels and 
standard CV risk factors for the entire study sample with or without adjustment for age and BMI.

\subsection{Within-age group correlation analyses}

When participants were divided by age, plasma fetuin-A was negatively correlated with $\mathrm{VO}_{2}$ max for both older (Figure $2 \mathrm{~A}$ ) and younger groups (Figure $\left.3 \mathrm{~A}\right)(\mathrm{P}<0.05)$. There was no significant relationship between plasma fetuin-A and insulin or HOMA-IR in the combined group; however, in the older subjects, plasma fetuin-A relationships demonstrated trends $(\mathrm{P}<0.06)$ for significance with plasma insulin and HOMA-IR (Figure 2B and 2C). In younger subjects, plasma fetuin-A was significantly related to blood pressure (Figure 3B and $3 \mathrm{C})$ and blood lipid variables (Figure 3D and 3E) $(\mathrm{P}<0.05)$.

\section{Discussion}

Our main findings are that plasma fetuin-A levels are (i) significantly lower in highly active men and inversely related to cardiorespiratory fitness $\left(\mathrm{VO}_{2} \mathrm{max}\right)$, (ii) not related to age, and (iii) related to various conventional $\mathrm{CV}$ risk factors in young and older men. These data indicate that physical activity may be an important factor in the maintenance of low plasma fetuin-A levels independent of age. These results, combined with others from the current literature showing fetuin-A to be associated with incident type 2 diabetes, provide evidence that plasma fetuin-A levels may be a novel factor through which regular endurance exercise acts to improve insulin sensitivity and reduce cardiometabolic risk.

\subsection{Relation of fetuin-A to $\mathrm{VO}_{2} \max$}

Currently, there is a paucity of data on CV fitness and plasma fetuin-A. Wilund et al. [22] reported a positive correlation between $\mathrm{VO}_{2}$ max and fetuin-A levels in older men, opposite of the current finding of a negative relationship. There are a number of possible reasons for the discrepancy between our data and this earlier study. Wilund et al. studied older high- and low-active men and women, and they found an association between $\mathrm{VO}_{2} \mathrm{max}$ and fetuin- $\mathrm{A}$ levels in men only. Their subjects were also slightly older than ours on average. We included a larger sample size ( $N=38$ vs. $N=25$ in [22]), and our subjects had wider ranges of age (18-77 vs. only $60+\mathrm{yr}$ ) and $\mathrm{VO}_{2}$ max values ( $20-70$ vs. $\left.20-45 \mathrm{ml} \cdot \mathrm{kg}^{-1} \cdot \mathrm{min}^{-1}\right)$. Therefore, our design may allow for a more complete characterization of the relationship between cardiorespiratory fitness and fetuin-A levels compared with Wilund et al. [22]. However, neither our data nor theirs can be regarded as definitive, given the cross-sectional study design and descriptive nature of both studies. It is clear that further work is needed to clarify the effect of long-term endurance exercise on fetuin-A levels, with special attention to establishing the direction of the relationship and determining the mechanisms underlying the apparent sex difference.

A number of investigators have argued that plasma fetuin-A is related to systemic inflammation. Wilund et al. [22] attributed the positive relation between $\mathrm{VO}_{2}$ max and fetuin-A levels in men to the anti-inflammatory effects of chronic exercise training. However, the literature relative to a potential role for plasma fetuin- $\mathrm{A}$ in inflammation is not clear. Fetuin-A has been positively associated with markers of inflammation such as Creactive protein $[8,17]$ and seems to have direct in vivo and in vitro pro-inflammatory effects [17], yet fetuin-A levels were found to be lower with chronic inflammation in patients undergoing hemodialysis [23]. Markers of inflammation were not measured in the current study; therefore, we cannot be certain that greater levels of inflammation in our chronic exercise groups were related to lower fetuin-A levels. However, much of the available data indicate that fetuin-A is an inhibitor of insulin signaling [7], consistent with the positive correlation of fetuin-A levels and incidence of type 2 diabetes and metabolic syndrome 
$[8,9]$. Thus, because these metabolic disorders are prevented or ameliorated with regular endurance exercise, we believe our negative correlation between $\mathrm{VO}_{2}$ max and fetuin- $\mathrm{A}$ is consistent with the well-known insulin-sensitizing effects of endurance training. Future studies are necessary to elucidate the interaction between exercise, inflammation, and fetuinA.

Our finding of a cross-sectional difference in fetuin-A levels among HI and LO groups is somewhat inconsistent with previous longitudinal data indicating that 3 months of thiazolidinedione treatment, but not endurance exercise training, can reduce fetuin-A levels in type 2 diabetes patients [24]. Thus, it is possible that thiazolidinedione may confer more rapid effects on fetuin-A levels than exercise training, but the effects of exercise training on fetuin-A levels have by no means been adequately addressed. There are at least two possible reasons for the discrepancy between our data and the exercise training data of Mori et al. [24]. First, our HI subjects had been performing regular vigorous endurance exercise for several years (3+ years for young $\mathrm{HI}$ and $30+$ years for older $\mathrm{HI}$ ), whereas the intervention period of Mori et al. [24] was only 3 months and the intensity and duration were substantially lower than that performed by our subjects. Second, we studied only healthy subjects with no history of CV or metabolic disease, while Mori et al. [24] examined patients with overt type 2 diabetes. Thus, it is apparent that disease status and the duration, intensity, and length of training are all important factors in determining the effect of longterm exercise on fetuin-A levels, but these issues will need to be systematically evaluated in future prospective trials.

\subsection{Fetuin-A, glucose metabolism, and body composition}

Fetuin-A is recognized as a liver-derived protein that is associated with insulin resistance and diabetes, presumably due to the affinity of fetuin-A for tyrosine kinase receptors. In particular, fetuin-A binding to insulin receptors in adipose and skeletal muscle tissues leads to decreased receptor phosphorylation and reduced downstream insulin signaling [7]. Exercise confers substantial metabolic benefits including pronounced skeletal muscle insulin sensitizing effects. Mechanisms include enhanced glucose disposal via improved glucose uptake and metabolism as well as increased expression and/or activity of proteins within the insulin signaling cascade [25]. Changes in hepatic function with exercise are less clear, with the majority of the benefit from exercise being attributed to decreased hepatic glucose production. Our data reveal that both younger and older low active men have higher circulating fetuin-A levels compared to chronically exercising men and in older men, fetuinA levels were related to plasma insulin and HOMA-IR. Physical inactivity may lead to increased hepatic fetuin-A production contributing to overall insulin resistance and type 2 diabetes [14].

Others have suggested that fetuin-A levels may be influenced by obesity. Recent data indicate that weight loss resulting from a combined dietary and physical activity intervention reduces fetuin-A levels in children with the metabolic syndrome [26]. Our data suggest that the effects of regular physical activity on fetuin-A occur independent of body fat, as there were differences in (i) fetuin-A levels between $\mathrm{HI}$ and $\mathrm{LO}$ groups of both age categories despite no within-age group differences in percent body fat, and (ii) body fatness between young and older LO men despite no differences in fetuin-A levels. In further support of these findings, Ix et al. [9] found that the relationship between fetuin-A and incident diabetes was only partially mediated by visceral adiposity in a retrospective case-cohort study of 70-79 year-olds, suggesting that body fat may not be the main factor relating fetuinA and diabetes. However, future studies are needed to define the individual and interactive effects of physical activity, body composition, and age on fetuin-A levels and the role of these effects in the development and prevention of diabetes and CV disease. Additionally, 
our data highlight the importance of including physical activity as a covariate (at minimum) in studies of age- and body composition-related differences in fetuin-A levels.

\subsection{Fetuin-A, blood pressure, and lipid variables}

There is good evidence that fetuin-A may interact with traditional CV risk factors. For example, high fetuin-A levels are associated with dyslipidemia and metabolic syndrome status [27], and combined weight loss and physical activity decreased fetuin-A levels in association with favorable changes in blood pressure in children with the metabolic syndrome [26]. Our data in young HI and LO individuals extend these findings by showing that fetuin-A levels are positively related to blood pressure and lipid variables in young healthy men. Importantly, fetuin-A levels are elevated in young men who would be considered very healthy with normal levels of conventional CV risk factors but who do not perform regular exercise. Thus, a reasonable working hypothesis from our observed correlations of fetuin-A with blood pressure and circulating lipids is that fetuin-A may interact with traditional risk factors early in the physical inactivity-related disease process.

\subsection{Limitations}

Our study is limited by a relatively small sample size and the cross-sectional design. The descriptive nature of our study prevented us from gaining any mechanistic insights into the involvement of fetuin-A in the beneficial effects of regular endurance exercise on CV and metabolic risk. Future studies will be required to determine the mechanisms underlying the differences in fetuin-A levels as well as the consequences of elevated fetuin-A levels at the cellular level. However, our study achieved its primary aim in documenting the associations of age and chronic exercise with fetuin-A levels. As the individuals included in our study were carefully recruited and characterized, we are confident that our study allows for conclusions about the effects of age and chronic exercise independent of BMI, body fat, and other potential confounding factors. In addition, our data provide important new information about the association between plasma fetuin-A levels and a number of conventional CV and metabolic risk factors in these well-characterized individuals.

\subsection{Conclusion}

In summary, fetuin-A is now recognized as an important independent contributor to metabolic and cardiovascular disorders [28]. Our data indicate that fetuin-A is related to cardiorespiratory fitness independent of age. Therefore, regular exercise throughout the lifetime may prevent increases in fetuin- $A$ in association with prevention of age- and inactivity-related cardiometabolic disorders. However, our findings must be interpreted with caution given the limitations discussed above and need confirmation in a future prospective trial.

\section{Acknowledgments}

N.T.J. and J.A.M. were supported by NIH Predoctoral Training Grant T32AG20068. The authors thank Drs. Barbara Albert and Thomas Obisesan for medical supervision of exercise testing.

\section{References}

1. Lloyd-Jones D, Adams R, Carnethon M, De Simone G, Ferguson TB, Flegal K, et al. Heart Disease and Stroke Statistics--2009 Update: A Report From the American Heart Association Statistics Committee and Stroke Statistics Subcommittee. Circulation 2009;119:e21-181. [PubMed: 19075105]

2. Kashyap SR, Defronzo RA. The insulin resistance syndrome: physiological considerations. Diab Vasc Dis Res 2007;4:13-9. [PubMed: 17469039] 
3. Braunwald E. Cardiovascular medicine at the turn of the millennium: triumphs, concerns, and opportunities. New Eng J Med 1997;337:1360-9. [PubMed: 9358131]

4. Helmrich SP, Ragland DR, Paffenbarger RS Jr. Prevention of non-insulin-dependent diabetes mellitus with physical activity. Med Sci Sports Exerc 1994;26:824-30. [PubMed: 7934754]

5. Mora S, Cook N, Buring JE, Ridker PM, Lee IM. Physical Activity and Reduced Risk of Cardiovascular Events: Potential Mediating Mechanisms. Circulation 2007;116:2110-8. [PubMed: 17967770]

6. Mozaffarian D, Wilson PWF, Kannel WB. Beyond Established and Novel Risk Factors: Lifestyle Risk Factors for Cardiovascular Disease. Circulation 2008;117:3031-8. [PubMed: 18541753]

7. Rauth G, Poschke O, Fink E, Eulitz M, Tippmer S, Kellerer M, et al. The nucleotide and partial amino acid sequences of rat fetuin. Identity with the natural tyrosine kinase inhibitor of the rat insulin receptor. Eur J Biochem 1992;204:523-9. [PubMed: 1371750]

8. Stefan N, Fritsche A, Weikert C, Boeing H, Joost HG, Haring HU, et al. Plasma fetuin-A levels and the risk of type 2 diabetes. Diabetes 2008;57:2762-7. [PubMed: 18633113]

9. Ix JH, Wassel CL, Kanaya AM, Vittinghoff E, Johnson KC, Koster A, et al. Fetuin-A and incident diabetes mellitus in older persons. JAMA 2008;300:182-8. [PubMed: 18612115]

10. Rittig K, Thamer C, Haupt A, Machann J, Peter A, Balletshofer B, et al. High plasma fetuin-A is associated with increased carotid intima-media thickness in a middle-aged population. Atherosclerosis 2009;207:341-2. [PubMed: 19615685]

11. Weikert C, Stefan N, Schulze MB, Pischon T, Berger K, Joost HG, et al. Plasma fetuin-a levels and the risk of myocardial infarction and ischemic stroke. Circulation 2008;118:2555-62. [PubMed: 19029462]

12. Fisher E, Stefan N, Saar K, Drogan D, Schulze MB, Fritsche A, et al. Association of AHSG gene polymorphisms with fetuin-A plasma levels and cardiovascular diseases in the EPIC-Potsdam study. Circ Cardiovasc Genet 2009;2:607-13. [PubMed: 20031641]

13. Church TS, Kuk JL, Ross R, Priest EL, Biltoft E, Blair SN. Association of cardiorespiratory fitness, body mass index, and waist circumference to nonalcoholic fatty liver disease. Gastroenterology 2006;130:2023-30. [PubMed: 16762625]

14. Stefan N, Hennige AM, Staiger H, Machann J, Schick F, Krober SM, et al. Alpha2-HeremansSchmid glycoprotein/fetuin-A is associated with insulin resistance and fat accumulation in the liver in humans. Diabetes Care 2006;29:853-7. [PubMed: 16567827]

15. Stefan N, Kantartzis K, Haring HU. Causes and metabolic consequences of Fatty liver. Endocr Rev 2008;29:939-60. [PubMed: 18723451]

16. Kantartzis K, Thamer C, Peter A, Machann J, Schick F, Schraml C, et al. High cardiorespiratory fitness is an independent predictor of the reduction in liver fat during a lifestyle intervention in non-alcoholic fatty liver disease. Gut 2009;58:1281-8. [PubMed: 19074179]

17. Hennige AM, Staiger H, Wicke C, Machicao F, Fritsche A, Haring HU, et al. Fetuin-A induces cytokine expression and suppresses adiponectin production. PLoS One 2008;3:e1765. [PubMed: 18335040]

18. Petersen AM, Pedersen BK. The anti-inflammatory effect of exercise. J Appl Physiol 2005;98:1154-62. [PubMed: 15772055]

19. Jenkins NT, Witkowski S, Spangenburg EE, Hagberg JM. Effects of acute and chronic endurance exercise on intracellular nitric oxide in putative endothelial progenitor cells: role of NAPDH oxidase. Am J Physiol Heart Circ Physiol 2009;297:H1798-H1805. [PubMed: 19717732]

20. Witkowski S, Lockard MM, Jenkins NT, Obisesan TO, Spangenburg EE, Hagberg JM. Relation of circulating progenitor cells to vascular function and oxidative stress with long term training and short term detraining in older men. Clin Sci (Lond). 2009 in press (Epub ahead of print).

21. Matthews DR, Hosker JP, Rudenski AS, Naylor BA, Treacher DF, Turner RC. Homeostasis model assessment: insulin resistance and beta-cell function from fasting plasma glucose and insulin concentrations in man. Diabetologia 1985;28:412-9. [PubMed: 3899825]

22. Wilund KR, Tomayko EJ, Evans EM, Kim K, Ishaque MR, Fernhall B. Physical activity, coronary artery calcium, and bone mineral density in elderly men and women: a preliminary investigation. Metabolism 2008;57:584-91. [PubMed: 18328364] 
23. Cozzolino M, Galassi A, Biondi ML, Turri O, Papagni S, Mongelli N, et al. Serum fetuin-A levels link inflammation and cardiovascular calcification in hemodialysis patients. Am J Nephrol 2006;26:423-9. [PubMed: 16968979]

24. Mori K, Emoto M, Araki T, Yokoyama H, Lee E, Teramura M, et al. Effects of pioglitazone on serum fetuin-A levels in patients with type 2 diabetes mellitus. Metabolism 2008;57:1248-52. [PubMed: 18702951]

25. Hawley JA, Lessard SJ. Exercise training-induced improvements in insulin action. Acta Physiol (Oxf) 2008;192:127-35. [PubMed: 18171435]

26. Reinehr T, Roth CL. Fetuin-A and its relation to metabolic syndrome and fatty liver disease in obese children before and after weight loss. J Clin Endocrinol Metab 2008;93:4479-85. [PubMed: 18728159]

27. Ix JH, Shlipak MG, Brandenburg VM, Ali S, Ketteler M, Whooley MA. Association between human fetuin-A and the metabolic syndrome: data from the Heart and Soul Study. Circulation 2006;113:1760-7. [PubMed: 16567568]

28. Mori K, Emoto M, Yokoyama H, Araki T, Teramura M, Koyama H, et al. Association of serum fetuin-A with insulin resistance in type 2 diabetic and nondiabetic subjects. Diabetes Care 2006;29:468. [PubMed: 16443916]

\section{Abbreviations}

CV

$\mathrm{VO}_{2} \max$

HOMA-IR

HI and LO, respectively
Cardiovascular

Maximal Oxygen Uptake

Homeostasis Model Assessment of Insulin Resistance

High- and Low-Active 


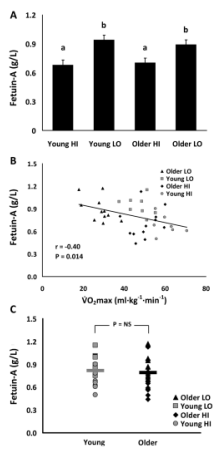

Figure 1.

(A) Influence of age and physical activity on plasma fetuin-A levels (data with like letters are not statistically different from each other at the $\alpha=0.05$ level); (B) relationship between maximal oxygen uptake $\left(\mathrm{VO}_{2} \mathrm{max}\right)$ and plasma fetuin-A levels; (C) age group comparison of and fetuin-A levels; symbols represent individual data points as in $B$, with solid bars indicating age group means. 


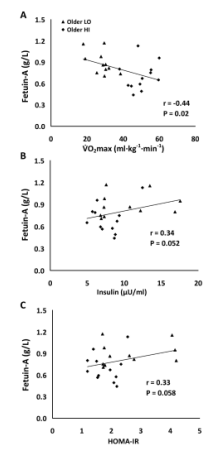

Figure 2.

Relation of $\mathrm{VO}_{2} \max (\mathrm{A})$, insulin (B), and homeostasis model assessment of insulin resistance (HOMA-IR) (C) to fetuin-A levels in older participants. 

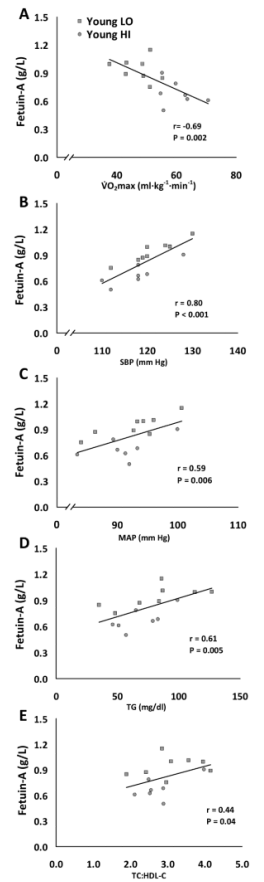

Figure 3.

Relation of $\mathrm{VO}_{2} \max (\mathrm{A})$, systolic blood pressure (SBP) (B), mean arterial pressure (MAP) (C), triglycerides (TG) (D), and the ratio of total cholesterol to high density lipoproteincholesterol (TC:HDL) (E) to fetuin-A levels in younger participants. 
Table 1

Descriptive characteristics (means $\pm \mathrm{SE}$ )

\begin{tabular}{|c|c|c|c|c|}
\hline & \multicolumn{2}{|c|}{ Young } & \multicolumn{2}{|c|}{ Older } \\
\hline & HI $(\mathbf{n}=7)$ & LO $(n=8)$ & HI $(n=12)$ & $\operatorname{LO}(n=11)$ \\
\hline Age (yr) & $24.4 \pm 1.5^{\mathrm{a}}$ & $24.6 \pm 1.7^{\mathrm{a}}$ & $61.7 \pm 1.6^{\mathrm{b}}$ & $64.5 \pm 1.5^{\mathrm{b}}$ \\
\hline BMI $\left(\mathrm{kg} / \mathrm{m}^{2}\right)$ & $24.4 \pm 1.5$ & $23.6 \pm 4.4$ & $22.9 \pm 0.8$ & $24.3 \pm 0.6$ \\
\hline Body fat (\%) & $14.3 \pm 2.2^{\mathrm{a}}$ & $14.8 \pm 2.4^{\mathrm{a}}$ & $18.0 \pm 1.3^{\mathrm{a}, \mathrm{b}}$ & $23.5 \pm 1.8^{b}$ \\
\hline Glucose (mg/dl) & $85.9 \pm 2.7^{\mathrm{a}}$ & $81.4 \pm 2.6^{\mathrm{a}}$ & $94.5 \pm 2.1^{\mathrm{a}, \mathrm{b}}$ & $99.0 \pm 2.6^{\mathrm{b}}$ \\
\hline Insulin $(\mu \mathrm{U} / \mathrm{ml})$ & $8.9 \pm 0.9$ & $7.2 \pm 0.5$ & $7.8 \pm 0.6$ & $10.4 \pm 1.2$ \\
\hline HOMA-IR & $1.9 \pm 0.2^{\mathrm{a}}$ & $1.5 \pm 0.1^{\mathrm{a}}$ & $1.8 \pm 0.1^{\mathrm{a}}$ & $2.6 \pm 0.3^{\mathrm{b}}$ \\
\hline $\mathrm{TC}(\mathrm{mg} / \mathrm{dl})$ & $145.9 \pm 8.0^{\mathrm{a}}$ & $146.8 \pm 8.7^{\mathrm{a}}$ & $199.1 \pm 8.9^{b}$ & $194.2 \pm 10.6^{\mathrm{b}}$ \\
\hline HDL-C (mg/dl) & $53.3 \pm 1.9^{\mathrm{a}}$ & $49.0 \pm 3.7^{\mathrm{a}}$ & $71.2 \pm 3.3^{b}$ & $51.0 \pm 4.6^{\mathrm{a}}$ \\
\hline LDL-C (mg/dl) & $78.9 \pm 8.1^{\mathrm{a}}$ & $81.5 \pm 7.8^{\mathrm{a}}$ & $114.8 \pm 8.4^{\mathrm{a}, \mathrm{b}}$ & $122.5 \pm 11.4^{\mathrm{b}}$ \\
\hline TC:HDL & $2.8 \pm 0.2^{\mathrm{a}}$ & $3.1 \pm 0.3^{\mathrm{a}}$ & $2.9 \pm 0.2^{\mathrm{a}}$ & $4.2 \pm 0.5^{\mathrm{b}}$ \\
\hline TG (mg/dl) & $68.6 \pm 7.3$ & $81.5 \pm 10.8$ & $66.2 \pm 8.4$ & $103.0 \pm 13.5$ \\
\hline $\mathrm{SBP}(\mathrm{mm} \mathrm{Hg})$ & $117 \pm 2^{\mathrm{a}}$ & $121 \pm 2^{a, b}$ & $122 \pm 3^{a, b}$ & $129 \pm 3^{b}$ \\
\hline $\mathrm{DBP}(\mathrm{mm} \mathrm{Hg})$ & $78 \pm 2$ & $79 \pm 2$ & $79 \pm 2$ & $85 \pm 2$ \\
\hline MAP (mm Hg) & $91 \pm 2^{\mathrm{a}}$ & $93 \pm 2^{\mathrm{a}}$ & $96 \pm 2^{a, b}$ & $101 \pm 2^{\mathrm{b}}$ \\
\hline $\mathrm{VO}_{2} \max (\mathrm{L} / \mathrm{min})$ & $4.8 \pm 0.2^{\mathrm{a}}$ & $3.6 \pm 0.2^{\mathrm{b}}$ & $3.5 \pm 0.2^{b}$ & $2.1 \pm 0.1^{\mathrm{c}}$ \\
\hline $\mathrm{VO}_{2} \max (\mathrm{ml} / \mathrm{kg} / \mathrm{min})$ & $60.4 \pm 2.2^{\mathrm{a}}$ & $47.3 \pm 2.0^{\mathrm{b}}$ & $50.0 \pm 1.9^{b}$ & $28.1 \pm 1.7^{\mathrm{c}}$ \\
\hline
\end{tabular}

Note: Values with like superscripts are not significantly different from each other at the $\alpha=0.05$ level.

Abbreviations: BMI, body mass index; HOMA-IR, homeostasis model assessment of insulin resistance; TC, total cholesterol; HDL-C, high density lipoprotein-cholesterol; LDL-C, low density lipoprotein-cholesterol; TC:HDL, TC to HDL-C ratio; TG, triglycerides; SBP, systolic blood pressure; DBP, diastolic blood pressure; MAP, mean arterial pressure; $\mathrm{VO}_{2}$ max, maximal oxygen uptake. 